$$
\begin{gathered}
\text { WHY } \\
\text { TRUST } \\
\text { SCIENCE } \\
?
\end{gathered}
$$




\section{The University Center for}

Human Values Series

\section{Stephen Macedo, Editor}

A list of titles in this series appears at the back of the book. 


$$
\begin{gathered}
\text { WHY } \\
\text { TRUST } \\
\text { SCIENCE } \\
\text { ? } \\
\text { NAOMI } \\
\text { ORESKES }
\end{gathered}
$$

PRINCETON UNIVERSITY PRESS

PRINCETON AND OXFORD 
Copyright (C) 2019 by Princeton University Press

Published by Princeton University Press

41 William Street, Princeton, New Jersey 08540

6 Oxford Street, Woodstock, Oxfordshire OX20 1TR

press.princeton.edu

All Rights Reserved

LCCN 2019937193

ISBN 9780691179001

British Library Cataloging-in-Publication Data is available

Editorial: Alison Kalett and Kristin Zodrow

Production Editorial: Sara Lerner

Text Design: Leslie Flis

Jacket Design: Amanda Weiss

Production: Jacqueline Poirier

Publicity: Sara Henning-Stout and Katie Lewis

Copyeditor: Brittany Micka-Foos

This book has been composed in Arno Pro with Gotham Display

Printed on acid-free paper. $\infty$

Printed in the United States of America

$\begin{array}{llllllllll}1 & 3 & 5 & 7 & 9 & 10 & 8 & 6 & 4\end{array}$ 
Trust, but verify.

- RONALD REAGAN 
